\title{
Adaptive Shape Control for Thermal Deformation of Membrane Mirror with In-plane PVDF Actuators
}

\author{
Yi-Fan Lu ${ }^{1 *} \mathbb{0}$, Hong-Hao Yue ${ }^{1}$, Zong-Quan Deng ${ }^{1}$ and Horn-Sen Tzou ${ }^{2}$
}

\begin{abstract}
Optical membrane mirrors are promising key components for future space telescopes. Due to their ultra-thin and high flexible properties, the surfaces of these membrane mirrors are susceptible to temperature variations. Therefore adaptive shape control of the mirror is essential to maintain the surface precision and to ensure its working performance. However, researches on modeling and control of membrane mirrors under thermal loads are sparse in open literatures. A $0.2 \mathrm{~m}$ diameter scale model of a polyimide membrane mirror is developed in this study. Three Polyvinylidene fluoride (PVDF) patches are laminated on the non-reflective side of the membrane mirror to serve as in-plane actuators. A new mathematical model of the piezoelectric actuated membrane mirror in multiple fields, (i.e., thermal, mechanical, and electrical field) is established, with which dynamic and static behaviors of the mirror can be analyzed. A closed-loop membrane mirror shape control system is set up and a surface shape control method based on an influence function matrix of the mirror is then investigated. Several experiments including surface displacement tracking and thermal deformation alleviation are performed. The deviations range from $15 \mu \mathrm{m}$ to $20 \mu \mathrm{m}$ are eliminated within $0.1 \mathrm{~s}$ and the residual deformation is controlled to micron level, which demonstrates the effectiveness of the proposed membrane shape control strategy and shows a satisfactory real-time performance. The proposed research provides a technological support and instruction for shape control of optical membrane mirrors.
\end{abstract}

Keywords: Adaptive shape control, Membrane mirror, Thermal deformation, PVDF, Influence function matrix

\section{Introduction}

Along with the development of deep space exploration, space communication, and earth observation technology, there is an increasing demand for high-resolution and wide-field optical mirrors used in space telescopes, radars, antennas, and energy collectors [1]. Large aperture mirrors have not only the ability of improving the clarity of a low-contrast image but also an increased field-of-vision that can greatly expand the range of earth observation [2]. Stable rigid materials are used as substrates for conventional large optical mirrors. These contribute to high costs, difficulty in processing, and long manufacturing cycles. Additionally, the dimensions of

\footnotetext{
*Correspondence: yf.lu@hit.edu.cn

${ }^{1}$ School of Mechatronics Engineering, Harbin Institute of Technology, Harbin 150001, China

Full list of author information is available at the end of the article
}

the rigid mirror are limited by its weight and the payload capacity of current launch vehicles. Therefore, a new technology called membrane optics has been proposed in recent years. A deployable membrane optical mirror has many advantages such as being ultra-lightweight and having a low areal density, which can potentially decrease the launching cost by reducing the launch vehicle size requirement. However, with an increase in mirror aperture and decrease in areal density, the stiffness drops, and the mirror becomes more vulnerable to outside interference. It is obvious that surface accuracy is of great importance to the performance of these mirrors. Large optical mirrors usually work in medium and geosynchronous earth orbits. Previous studies have demonstrated that the main cause of surface shape errors in these orbits is temperature variations [3]. Thus, the development of methods for closed-loop surface shape control
Springer Open

(c) Author(s) 2018. This article is distributed under the terms of the Creative Commons Attribution 4.0 International License (http://creativecommons.org/licenses/by/4.0/), which permits unrestricted use, distribution, and reproduction in any medium, provided you give appropriate credit to the original author(s) and the source, provide a link to the Creative Commons license, and indicate if changes were made. 
of membrane mirrors under thermal loads is of considerable significance.

Many flatness control methods have been explored including in-plane patching-on/embedding-in, boundary actuation, and out-of-plane electrostatic actuation to ensure the surface accuracy of membrane mirrors. Various smart materials such as shape-memory alloys (SMAs) [4], polyvinylidene fluoride (PVDF) films [5-7], piezoelectric polymer actuators [8], macrofiber composites $[9,10]$, and microelectromechanical system transducers [11] have been used to control the surface shape of membrane structures. A parabolic deformable mirror with a piezoelectric thin film was studied and controlled based on an in-plane actuation strategy by Maji et al. [12] and the active control precision proved to reach the micron level. Errico et al. [13] developed a hybrid control method combining boundary piezoelectric tension with transverse electrostatic actuation. A 6-inch-thick membrane mirror was built and electrostatic pressure was used to pull the nominally flat mirror to a $32 \mathrm{~m}$ radius of curvature and to adaptively correct its aberrations. A series of tests on membrane shape control with SMA wires were undertaken by the Canadian Space Agency. A generic algorithm-based control system was developed and tested on a square membrane by Peng et al. [14-17] and Wang et al. [18-20] investigated active flatness control of membrane structures under thermal and mechanical loads with SMA actuators. Furthermore, Shan et al. [21] in York University developed a photogrammetry software to measure the flatness of a membrane structure that actively boundary controlled by SMA wires. Mathematical models of the circular membrane mirror were studied by Jenkins and thermal load effects were taken into account later [22, 23]. The wrinkling of the membrane structure according to the thermoelastic boundary conditions was discussed [24], and an adaptive control method was used to improve the surface accuracy of the membrane reflector [25]. Blandino [26, 27] and Hornig et al. [28] also surveyed and experimented on the wrinkling of membrane structures due to mechanical and thermal loads. A unimorph piezoelectric-actuated membrane mirror model was established by Shepherd [29], and a closed-loop shape control that reached the micron level was achieved. Laslandes et al. [30] investigated actuator optimization of an ultrathin deformable mirror, whereas Rausch et al. [31] conducted a series of tests for a piezoelectric-actuated deformable mirror.

In this study, a mathematical model of a PVDF laminated membrane mirror in thermal, mechanical, and electrical field is established and expressions of the membrane forces and bending moments are derived. A 0.2 $\mathrm{m}$ diameter in-plane-actuated membrane mirror with laminated PVDF actuators is constructed for testing.
High-precision laser displacement sensors are used to measure the surface deviation of the membrane and serve as feedback for the closed-loop shape control system. An experimental platform for surface shape control of the mirror is setup and several experiments are performed to determine the efficiency of our mirror shape control methodology.

\section{Modeling of the Membrane Mirror}

This section describes the study of a PVDF laminated membrane mirror consisting of two layers (i.e., a polyimide substrate layer and a PVDF actuator layer). The scale model of the mirror in the polar coordinate system is presented in Figure 1, where $h_{s}$ and $h_{p}$ are the thickness of the substrate and actuator layer respectively. Based on the Kirchhoff-Love theory for thin shells, the mirror model can be simplified from the fundamental form and governing equations to a generic double curvature shell with specified two Lamé parameters and two radii of curvature [32, 33].

For the flat circular membrane mirror model, the two Lamé parameters are $A_{1}=1$ and $A_{2}=r$; and the two radii of curvature are $R_{1}=R_{2}=\infty$. With the four parameters, one can simplify the double-curvature shell dynamic equations to the governing equations of the mirror model:

$$
\begin{aligned}
& \frac{\partial N_{1}}{\partial r}+\frac{1}{r} \frac{\partial N_{6}}{\partial \theta}+\frac{N_{1}-N_{2}}{r}=\rho h \frac{\partial^{2} u}{\partial t^{2}}-q_{1}, \\
& \frac{\partial N_{6}}{\partial r}+\frac{1}{r} \frac{\partial N_{2}}{\partial \theta}+\frac{2 N_{6}}{r}=\rho h \frac{\partial^{2} v}{\partial t^{2}}-q_{2}, \\
& \frac{\partial Q_{1}}{\partial r}+\frac{1}{r} \frac{\partial Q_{2}}{\partial \theta}+\frac{Q_{1}}{r}+\frac{1}{r} \frac{\partial}{\partial \theta}\left(\frac{N_{2}}{r} \frac{\partial w}{\partial \theta}+N_{6} \frac{\partial w}{\partial r}\right) \\
& \quad+\frac{1}{r} \frac{\partial}{\partial r}\left(r N_{1} \frac{\partial w}{\partial r}+N_{6} \frac{\partial w}{\partial \theta}\right)=\rho h \frac{\partial^{2} w}{\partial t^{2}}-q_{3}, \\
& \frac{\partial M_{6}}{\partial r}+\frac{1}{r} \frac{\partial M_{2}}{\partial \theta}+\frac{2 M_{6}}{r}-Q_{2}=0, \\
& \frac{\partial M_{1}}{\partial r}+\frac{1}{r} \frac{\partial M_{6}}{\partial \theta}+\frac{M_{1}-M_{2}}{r}-Q_{1}=0 .
\end{aligned}
$$

Substituting Eq. (4) and Eq. (5) into Eq. (3) to eliminate $Q_{1}$ and $Q_{2}$ yields

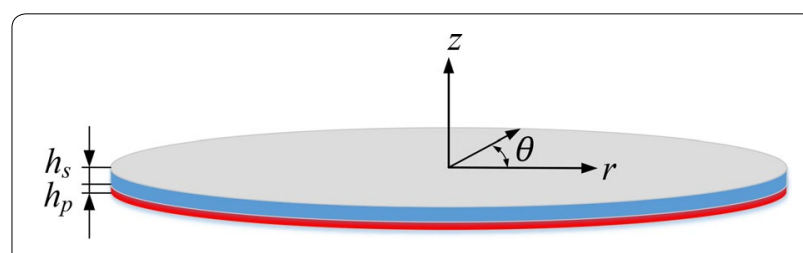

Figure 1 Configuration of a PVDF laminated membrane mirror 


$$
\begin{array}{r}
\frac{\partial^{2} M_{1}}{\partial r^{2}}+\frac{2}{r} \frac{\partial M_{1}}{\partial r}+\frac{1}{r^{2}} \frac{\partial^{2} M_{2}}{\partial \theta^{2}}-\frac{1}{r} \frac{\partial M_{2}}{\partial r}+\frac{2}{r} \frac{\partial^{2} M_{6}}{\partial r \partial \theta} \\
+\frac{2}{r^{2}} \frac{\partial M_{6}}{\partial \theta}+N_{1} \frac{\partial^{2} w}{\partial r^{2}}+N_{2}\left(\frac{1}{r} \frac{\partial w}{\partial r}+\frac{1}{r^{2}} \frac{\partial^{2} w}{\partial \theta^{2}}\right) \\
+2 N_{6}\left(\frac{1}{r} \frac{\partial^{2} w}{\partial r \partial \theta}-\frac{1}{r^{2}} \frac{\partial w}{\partial \theta}\right)=\rho h \frac{\partial^{2} w}{\partial t^{2}}-q_{3}
\end{array}
$$

where $q_{i}$ is the distributed load component in the $i$ th direction per unit area; $N_{i}$ is the membrane force per unit length; $M_{i}$ is the bending moment per unit length; $Q_{i}$ is the shear force per unit length; and $u, v$, and $w$ are the displacements in the radial, angular, and transverse directions. Considering that the size in the thickness direction of the mirror is usually much less than that in the other two directions, only the vibration and deformation in the transverse direction are studied. Thus, it is assumed that $\rho h \frac{\partial^{2} u}{\partial t^{2}}=\rho h \frac{\partial^{2} v}{\partial t^{2}}=0$. In a real application in space, $q_{3}$ denotes the microgravity and solar radiation pressure component in the transverse direction, while $q_{1}$ and $q_{2}$ are ignored. Note that

$$
\rho h=\sum_{k=1}^{N} \rho_{k} h_{k}
$$

where $\rho_{k}$ and $h_{k}$ are the mass density and thickness of the $k$ th layer respectively, and $N$ is the total layer number. This membrane mirror model consists of a substrate layer and an actuator layer; thus, $N=2$.

Utilizing the planar stress assumption that the principal stress normal to the surface is much smaller than the in-plane stresses, one may write $\sigma_{z z}=0$ and define the constitutive relationship of each layer:

$$
\begin{gathered}
\left(\begin{array}{c}
\sigma_{r r} \\
\sigma_{\theta \theta} \\
\sigma_{r \theta}
\end{array}\right)=\boldsymbol{c}^{E}\left(\begin{array}{c}
\varepsilon_{r r} \\
\varepsilon_{\theta \theta} \\
\varepsilon_{r \theta}
\end{array}\right)=\left(\begin{array}{lll}
c_{11} & c_{12} & c_{16} \\
c_{21} & c_{22} & c_{26} \\
c_{61} & c_{62} & c_{66}
\end{array}\right) . \\
\left(\left(\begin{array}{l}
e_{1} \\
e_{2} \\
\gamma_{6}
\end{array}\right)+z\left(\begin{array}{l}
k_{1} \\
k_{2} \\
k_{6}
\end{array}\right)\right),
\end{gathered}
$$

where the membrane strains $e_{i}$ and $\gamma_{i}$ are defined as

$$
\begin{aligned}
& e_{1}=\frac{\partial u}{\partial r}+\frac{1}{2}\left(\frac{\partial w}{\partial r}\right)^{2}, \\
& e_{2}=\frac{1}{r} \frac{\partial v}{\partial \theta}+\frac{u}{r}+\frac{1}{2 r^{2}}\left(\frac{\partial w}{\partial \theta}\right)^{2}, \\
& \gamma_{6}=\frac{\partial v}{\partial r}-\frac{v}{r}+\frac{1}{r} \frac{\partial u}{\partial \theta}+\frac{1}{r} \frac{\partial w}{\partial r} \frac{\partial w}{\partial \theta} .
\end{aligned}
$$

The bending strains $k_{i}$ are defined as

$$
\begin{aligned}
& k_{1}=-\frac{\partial^{2} w}{\partial r^{2}}, \\
& k_{2}=-\frac{1}{r^{2}} \frac{\partial^{2} w}{\partial \theta^{2}}-\frac{1}{r} \frac{\partial w}{\partial r}, \\
& k_{6}=\frac{2}{r^{2}} \frac{\partial w}{\partial \theta}-\frac{2}{r} \frac{\partial^{2} w}{\partial r \partial \theta} .
\end{aligned}
$$

For each transversely isotropic layer with Young's modulus $E$ and Poisson's ratio $v$, the constitutive stiffness matrix is

$\boldsymbol{c}^{E}=\left(\begin{array}{lll}c_{11} & c_{12} & c_{16} \\ c_{21} & c_{22} & c_{26} \\ c_{61} & c_{62} & c_{66}\end{array}\right)=\frac{E}{1-v^{2}}\left(\begin{array}{ccc}1 & v & 0 \\ v & 1 & 0 \\ 0 & 0 & (1-v) / 2\end{array}\right)$.

The mechanical membrane forces $N_{i}^{m}$ and bending moments $M_{i}^{m}$ for each layer are defined as

$$
\left(\begin{array}{c}
N_{1}^{m} \\
N_{2}^{m} \\
N_{6}^{m} \\
M_{1}^{m} \\
M_{2}^{m} \\
M_{6}^{m}
\end{array}\right)=\int_{z}\left(\begin{array}{c}
\sigma_{r r} \\
\sigma_{\theta \theta} \\
\sigma_{r \theta} \\
z \sigma_{r r} \\
z \sigma_{\theta \theta} \\
z \sigma_{r \theta}
\end{array}\right) \mathrm{d} z .
$$

Combining Eq. (8) and Eq. (16), including the thermal, piezoelectric, and tension force of the laminated membrane mirror will result in

$$
\begin{gathered}
\left(\begin{array}{l}
N_{1} \\
N_{2} \\
N_{6} \\
M_{1} \\
M_{2} \\
M_{6}
\end{array}\right)=\left(\begin{array}{l}
N_{1}^{m} \\
N_{2}^{m} \\
N_{6}^{m} \\
M_{1}^{m} \\
M_{2}^{m} \\
M_{6}^{m}
\end{array}\right)+\left(\begin{array}{c}
-N_{1}^{P}-N^{T}+N^{0} \\
-N_{2}^{P}-N^{T}+N^{0} \\
-N_{6}^{P} \\
-M_{1}^{P}-M^{T} \\
-M_{2}^{P}-M^{T} \\
-M_{6}^{P}
\end{array}\right)= \\
\left(\begin{array}{ll}
\boldsymbol{A}_{\boldsymbol{i j}} & \boldsymbol{B}_{\boldsymbol{i j}} \\
\boldsymbol{B}_{\boldsymbol{i j}} & \boldsymbol{D}_{\boldsymbol{i j}}
\end{array}\right)\left(\begin{array}{l}
e_{1} \\
e_{2} \\
\gamma_{6} \\
k_{1} \\
k_{2} \\
k_{6}
\end{array}\right)+\left(\begin{array}{c}
-N_{1}^{P}-N^{T}+N^{0} \\
-N_{2}^{P}-N^{T}+N^{0} \\
-N_{6}^{P} \\
-M_{1}^{P}-M^{T} \\
-M_{2}^{P}-M^{T} \\
-M_{6}^{P}
\end{array}\right),
\end{gathered}
$$

where the superscripts $P$ and $T$ denote the piezoelectric and thermal terms respectively, and $N^{0}$ denotes the membrane tension on the boundary of the mirror. Note that the thermal loads have no effect on the shear force and moment; external boundary tensioning will not induce shear forces and extra bending moments.

For an $N$ layer laminated structure with total thickness $h$, where the $k$ th laminae is located between the two 
planes $z=z_{k}$ and $z=z_{k+1}$, the following expressions can be derived [34]:

$$
\begin{aligned}
& A_{i j}=\sum_{k=1}^{N}\left(c_{i j}\right)_{k}\left(z_{k+1}-z_{k}\right), i, j=1,2,6, \\
& B_{i j}=\frac{1}{2} \sum_{k=1}^{N}\left(c_{i j}\right)_{k}\left(z_{k+1}^{2}-z_{k}^{2}\right), i, j=1,2,6, \\
& D_{i j}=\frac{1}{3} \sum_{k=1}^{N}\left(c_{i j}\right)_{k}\left(z_{k+1}^{3}-z_{k}^{3}\right), i, j=1,2,6 .
\end{aligned}
$$

In order to address the coupling terms in matrix $\boldsymbol{A}_{i j}$, $\boldsymbol{B}_{i j}$, and $\boldsymbol{D}_{i j}$, the neutral axis of the composite mirror is defined in Figure 2.

Where in Figure 2, $h_{s}$ is the thickness of the substrate, $h_{p}$ is the thickness of the PVDF actuator, and $d_{N A}$ is the distance between the bottom surface and the neutral axis. $d_{N A}$ can be calculated by summing up the in-plane stresses over the total thickness and equating the sum to zero:

$\int_{-d_{N A}}^{-d_{N A}+h_{p}} \frac{E_{p}}{1+v_{p}} z \mathrm{~d} z+\int_{-d_{N A}+h_{p}}^{-d_{N A}+h_{p}+h_{s}} \frac{E_{s}}{1+v_{s}} z \mathrm{~d} z=0$,

where $E_{p}$ and $E_{s}$ are the Young's modulus of PVDF and substrate; $v_{p}$ and $v_{s}$ are the Poisson's ratio of PVDF and substrate respectively. Solving Eq. (21) for $d_{N A}$ yields

$$
d_{N A}=\frac{1}{2} \frac{E_{p} h_{p}^{2}+\delta E_{s}\left(2 h_{p} h_{s}+h_{s}^{2}\right)}{E_{p} h_{p}+\delta E_{s} h_{s}},
$$

where

$$
\delta \equiv \frac{1+v_{p}}{1+v_{s}} .
$$

In general, the material densities of the two layers are assumed to be similar and the mirror is constructed such that $h_{p}=d_{N A}$. From Eq. (22), one can derive the relationship below:

$$
h_{s}=h_{p} \sqrt{\frac{E_{p}}{\delta E_{s}}} .
$$

Next, we define two new parameters $\alpha$ and $\beta$ as

$$
\begin{gathered}
\alpha \equiv v_{s}-v_{p}, \\
\beta \equiv \frac{1-v_{p}}{1-v_{s}} .
\end{gathered}
$$

Solving Eqs. (18)-(20) with Eq. (15) and substituting $\alpha$ and $\beta$ result in

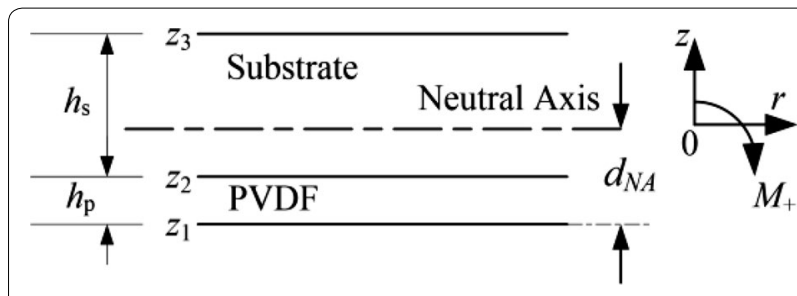

Figure 2 Neutral axis of the mirror

$$
\begin{aligned}
& \boldsymbol{A}_{i j}=\frac{E_{p} h_{p}}{1-v_{p}^{2}}\left\{\left(1+\frac{h_{p}}{h_{s}} \beta\right) \boldsymbol{E}_{i j}+\alpha \frac{h_{p}}{h_{s}} \beta \tilde{\boldsymbol{E}}_{i j}\right\}, \\
& \boldsymbol{B}_{i j}=\frac{1}{2} \frac{E_{p} h_{p}^{2}}{1-v_{p}^{2}}\left\{(\beta-1) \boldsymbol{E}_{i j}+\alpha \beta \tilde{\boldsymbol{E}}_{i j}\right\}, \\
& \boldsymbol{D}_{\boldsymbol{i} j}=\frac{1}{3} \frac{E_{p} h_{p}^{3}}{1-v_{p}^{2}}\left\{\left(1+\frac{h_{s}}{h_{p}} \beta\right) \boldsymbol{E}_{\boldsymbol{i}}+\alpha \frac{h_{s}}{h_{p}} \beta \tilde{\boldsymbol{E}}_{\boldsymbol{i}}\right\},
\end{aligned}
$$

where

$$
\begin{aligned}
\boldsymbol{E}_{i j} & =\left(\begin{array}{ccc}
1 & v_{p} & 0 \\
v_{p} & 1 & 0 \\
0 & 0 & \left(1-v_{p}\right) / 2
\end{array}\right), \\
\tilde{\boldsymbol{E}}_{i j} & =\left(\begin{array}{ccc}
0 & 1 & 0 \\
1 & 0 & 0 \\
0 & 0 & -1 / 2
\end{array}\right) .
\end{aligned}
$$

When the Poisson's ratio of two materials are similar, i.e., $\alpha \approx 0, \delta \approx 0$, and $\beta \approx 1$, Eqs. (27)-(29) can be simplified to

$$
\begin{aligned}
& \boldsymbol{A}_{\boldsymbol{i j}}=A^{E} \boldsymbol{E}_{\boldsymbol{i j}}, \\
& \boldsymbol{B}_{\boldsymbol{i j}}=(\mathbf{0})_{3 \times 3}, \\
& \boldsymbol{D}_{\boldsymbol{i j}}=D^{E} \boldsymbol{E}_{\boldsymbol{i j}},
\end{aligned}
$$

where

$$
A^{E}=\frac{E_{p} h_{p}}{1-v_{p}^{2}}\left(1+\frac{h_{p}}{h_{s}}\right)
$$

$$
D^{E}=\frac{1}{3} \frac{E_{p} h_{p}^{3}}{1-v_{p}^{2}}\left(1+\frac{h_{s}}{h_{p}}\right) .
$$

Considering the ultrathin property of the deformable membrane mirror, the axial temperature gradient can be ignored compared with the radial temperature 
distribution, which mainly induces thermal stress and strain in the optical system. Thus, the temperature field on the mirror is defined as $T=T(r, \theta)$. The thermally induced force and moment are calculated as

$$
\begin{aligned}
N^{T} & =\frac{E \alpha}{1-v} \int_{z}\left(T-T_{0}\right) \mathrm{d} z \\
& =\left(\frac{E_{s} h_{s} \alpha_{s}}{1-v_{s}}+\frac{E_{p} h_{p} \alpha_{p}}{1-v_{p}}\right)\left(T-T_{0}\right), \\
M^{T} & =\frac{E \alpha}{1-v} \int_{z} z\left(T-T_{0}\right) \mathrm{d} z \\
& =\frac{1}{2}\left(\frac{E_{s} h_{s}^{2} \alpha_{s}}{1-v_{s}}-\frac{E_{p} h_{p}^{2} \alpha_{p}}{1-v_{p}}\right)\left(T-T_{0}\right),
\end{aligned}
$$

where $\alpha_{p}$ and $\alpha_{s}$ are the thermal expansion coefficients of PVDF and substrate respectively. Based on the piezoelectric constitutive equations [20], the piezoelectric induced forces and moments are derived as

$$
\begin{aligned}
& N_{1}^{P}=V_{3}\left(\cos ^{2} \theta e_{31}+\sin ^{2} \theta e_{32}\right) \\
& N_{2}^{P}=V_{3}\left(\sin ^{2} \theta e_{31}+\cos ^{2} \theta e_{32}\right) \\
& N_{6}^{P}=V_{3}\left(\cos \theta \sin \theta e_{31}-\cos \theta \sin \theta e_{32}\right) \\
& M_{1}^{P}=-\frac{1}{2} V_{3}\left(\cos ^{2} \theta e_{31}+\sin ^{2} \theta e_{32}\right) h_{p} \\
& M_{2}^{P}=-\frac{1}{2} V_{3}\left(\sin ^{2} \theta e_{31}+\cos ^{2} \theta e_{32}\right) h_{p} \\
& M_{6}^{P}=-\frac{1}{2} V_{3}\left(\cos \theta \sin \theta e_{31}-\cos \theta \sin \theta e_{32}\right) h_{p}
\end{aligned}
$$

where $V_{3}$ is the applied voltage in the $z$-direction, and $e_{31}$ and $e_{32}$ are the piezoelectric coupling coefficients of PVDF.

Substituting Eq. (17) into Eq. (6) and simplifying it with Eqs. (32)-(34) yields

$$
\begin{aligned}
& D^{E} \nabla^{4} w+\rho h \frac{\partial^{2} w}{\partial t^{2}}=N_{1} \frac{\partial^{2} w}{\partial r^{2}}+ \\
& N_{2}\left(\frac{1}{r} \frac{\partial w}{\partial r}+\frac{1}{r^{2}} \frac{\partial^{2} w}{\partial \theta^{2}}\right)+2 N_{6}\left(\frac{1}{r} \frac{\partial^{2} w}{\partial r \partial \theta}-\frac{1}{r^{2}} \frac{\partial w}{\partial \theta}\right)- \\
& \left(\frac{\partial^{2}}{\partial r^{2}}+\frac{2}{r} \frac{\partial}{\partial r}\right) M_{1}^{P}-\left(\frac{1}{r^{2}} \frac{\partial^{2}}{\partial \theta^{2}}-\frac{1}{r} \frac{\partial}{\partial r}\right) M_{2}^{P}- \\
& \left(\frac{2}{r} \frac{\partial^{2}}{\partial r \partial \theta}+\frac{2}{r^{2}} \frac{\partial}{\partial \theta}\right) M_{6}^{P}-\nabla^{2} M^{T}+q_{3} .
\end{aligned}
$$

In order to eliminate $N_{i}$ from Eq. (45), the stress function $\phi=\phi(r, \theta, t)$ is introduced; this satisfies exactly Eqs. (1) and (2) defined by

$$
\begin{aligned}
& N_{1}=\frac{1}{r} \frac{\partial \phi}{\partial r}+\frac{1}{r^{2}} \frac{\partial^{2} \phi}{\partial \theta^{2}}, \\
& N_{2}=\frac{\partial^{2} \phi}{\partial r^{2}}, \\
& N_{6}=-\frac{1}{r} \frac{\partial^{2} \phi}{\partial r \partial \theta}+\frac{1}{r^{2}} \frac{\partial \phi}{\partial \theta} .
\end{aligned}
$$

Note that in-plane inertia are neglected from Eqs. (1) and (2). Substituting $N_{i}$ from Eqs. (46)-(48) into Eq. (45), yields

$$
\begin{aligned}
D^{E} \nabla^{4} w & +\rho h \frac{\partial^{2} w}{\partial t^{2}} \\
= & \left(\frac{1}{r} \frac{\partial \phi}{\partial r}+\frac{1}{r^{2}} \frac{\partial^{2} \phi}{\partial \theta^{2}}\right) \frac{\partial^{2} w}{\partial r^{2}}+\frac{\partial^{2} \phi}{\partial r^{2}}\left(\frac{1}{r} \frac{\partial w}{\partial r}+\frac{1}{r^{2}} \frac{\partial^{2} w}{\partial \theta^{2}}\right) \\
& +\left(-\frac{2}{r} \frac{\partial^{2} \phi}{\partial r \partial \theta}+\frac{2}{r^{2}} \frac{\partial \phi}{\partial \theta}\right)\left(\frac{1}{r} \frac{\partial^{2} w}{\partial r \partial \theta}-\frac{1}{r^{2}} \frac{\partial w}{\partial \theta}\right) \\
& -\left(\frac{\partial^{2}}{\partial r^{2}}+\frac{2}{r} \frac{\partial}{\partial r}\right) M_{1}^{P}-\left(\frac{1}{r^{2}} \frac{\partial^{2}}{\partial \theta^{2}}-\frac{1}{r} \frac{\partial}{\partial r}\right) M_{2}^{P} \\
& -\left(\frac{2}{r} \frac{\partial^{2}}{\partial r \partial \theta}+\frac{2}{r^{2}} \frac{\partial}{\partial \theta}\right) M_{6}^{P}-\nabla^{2} M^{T}+q_{3} .
\end{aligned}
$$

Eq. (49) is the governing equation in the $z$-direction of the laminated membrane mirror and it needs to be augmented with the compatibility equation. Eliminating $u$ and $v$ from Eqs. (9)-(11) yields the following compatibility equation:

$$
\begin{aligned}
& \frac{\partial^{2} e_{1}}{\partial \theta^{2}}-\frac{\partial^{2}\left(r \gamma_{6}\right)}{\partial r \partial \theta}+\frac{\partial}{\partial r}\left(r^{2} \frac{\partial e_{2}}{\partial r}\right)-r \frac{\partial e_{1}}{\partial r} \\
& =\left(\frac{\partial^{2} w}{\partial r \partial \theta}-\frac{1}{r} \frac{\partial w}{\partial \theta}\right)^{2}-\frac{\partial^{2} w}{\partial r^{2}}\left(r \frac{\partial w}{\partial r}+\frac{\partial^{2} w}{\partial \theta^{2}}\right) .
\end{aligned}
$$

Solving Eq. (17) for $e_{1}, e_{2}$, and $\gamma_{6}$ yields

$$
\begin{aligned}
e_{1}= & \frac{h_{s}}{E_{P} h_{p}\left(h_{s}+h_{p}\right)}\left\{\left(N_{1}-v_{p} N_{2}\right)+\left(N_{1}^{P}-v_{p} N_{2}^{P}\right)\right. \\
& \left.+N^{T}\left(1-v_{p}\right)-N^{0}\left(1-v_{p}\right)\right\}, \\
e_{2}= & \frac{h_{s}}{E_{P} h_{p}\left(h_{s}+h_{p}\right)}\left\{\left(N_{2}-v_{p} N_{1}\right)+\left(N_{2}^{P}-v_{p} N_{1}^{P}\right)\right. \\
& \left.+N^{T}\left(1-v_{p}\right)-N^{0}\left(1-v_{p}\right)\right\}, \\
& \gamma_{6}=\frac{h_{s}}{E_{P} h_{p}\left(h_{s}+h_{p}\right)} 2\left(1+v_{p}\right)\left(N_{6}+N_{6}^{P}\right) .
\end{aligned}
$$




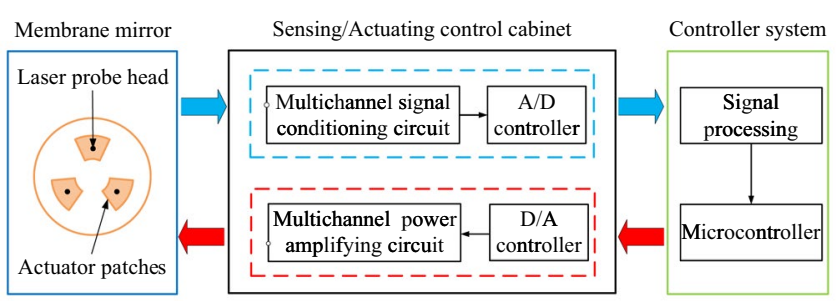

Figure 3 Block diagram of the adaptive shape control system

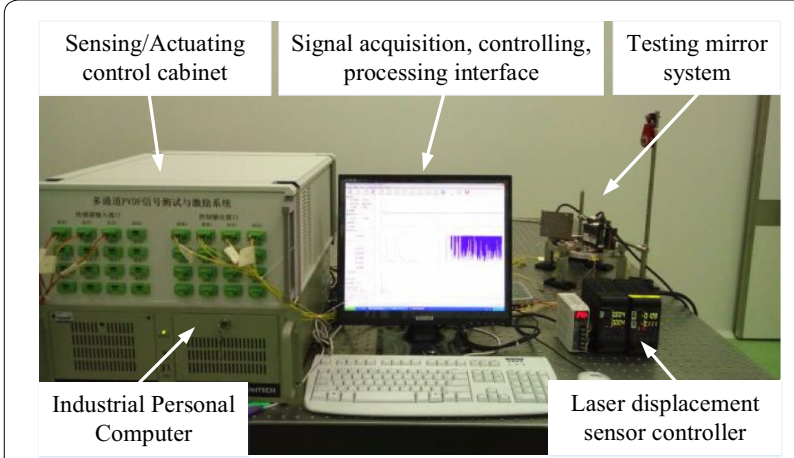

Figure 4 Experimental setup for active control system of a laminated membrane mirror

Substituting Eqs. (51)-(53) into Eq. (50) and using Eqs. (46)-(48) to simplify the result, we obtain

$$
\begin{aligned}
\nabla^{4} \phi= & E_{p} h_{p}\left(1+\frac{h_{p}}{h_{s}}\right)\left[\left(\frac{1}{r} \frac{\partial^{2} w}{\partial r \partial \theta}-\frac{1}{r^{2}} \frac{\partial w}{\partial \theta}\right)^{2}\right. \\
& \left.-\frac{\partial^{2} w}{\partial r^{2}}\left(\frac{1}{r} \frac{\partial w}{\partial r}+\frac{1}{r^{2}} \frac{\partial^{2} w}{\partial \theta^{2}}\right)\right] \\
& -\left(1-v_{p}\right) \nabla^{2}\left(N^{T}-N^{0}\right) \\
& -\left(-v_{p} \frac{\partial^{2}}{\partial r^{2}}-\frac{1+2 v_{p}}{r} \frac{\partial}{\partial r}+\frac{1}{r^{2}} \frac{\partial^{2}}{\partial \theta^{2}}\right) N_{1}^{P} \\
& -\left(\frac{\partial^{2}}{\partial r^{2}}+\frac{2+v_{p}}{r} \frac{\partial}{\partial r}-\frac{v_{p}}{r^{2}} \frac{\partial^{2}}{\partial \theta^{2}}\right) N_{2}^{P} \\
& +2\left(1+v_{p}\right)\left(\frac{1}{r^{2}} \frac{\partial}{\partial \theta}+\frac{1}{r} \frac{\partial^{2}}{\partial r \partial \theta}\right) N_{6}^{P},
\end{aligned}
$$

Eqs. (49) and (54) are the starting equations for analyzing the dynamics and statics of the piezoelectric laminated membrane mirror.

\section{Experimental Setup}

An adaptive membrane shape control experimental platform is established as described in this section. The experimental setup is composed of two systems: a circular membrane mirror with fixed boundary condition

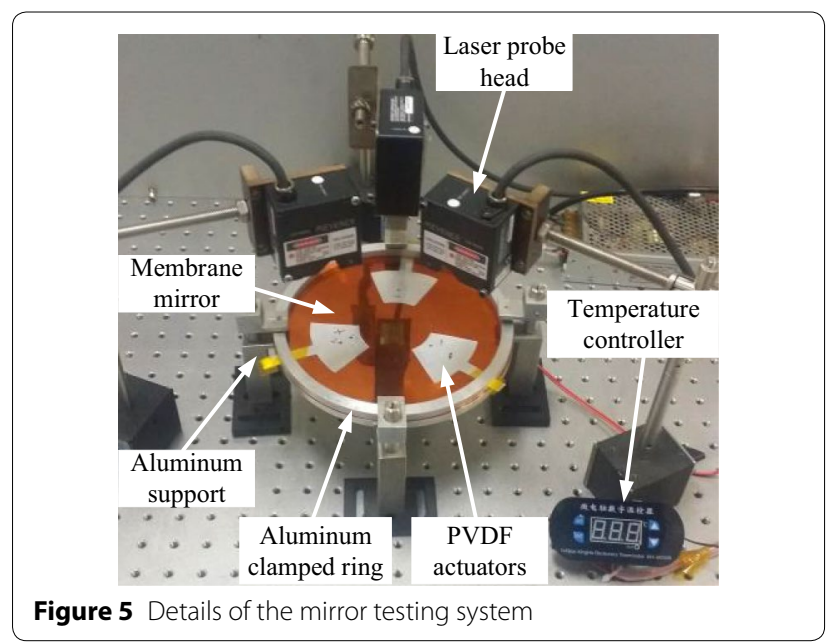

and an active control system. Three laser displacement sensors produced by Keyence Corporation are used for measuring the surface deviation of the mirror for feedback shape control, and three PVDF patches are laminated to the nonreflective side of the mirror to serve as actuators for surface shape control.

Figure 3 illustrates the system block diagram of the PVDF laminated membrane mirror in the laboratory experiments. A PXI-6284 A/D (analog-to-digital) card and a PXI-6723 D/A card provided by NI-DAQ (National Instruments) are used in the experimental setup for data acquisition of the sensors and outputs of the control signals. A high-performance embedded microcontroller PXI-8106 supplied by National Instruments is selected.

The adaptive shape control testing platform of the piezoelectric laminated membrane mirror is shown in Figure 4 . The hardware consists of a mirror testing system, a signal acquisition and controlling processing interface, a sensing/ actuating control cabinet, an industrial personal computer, and a laser displacement sensor controller. The sensing/actuating control cabinet contains a multichannel sensing signal conditioning circuit, an A/D conversion module, a D/A conversion module, a multichannel voltage amplifying circuit, and some other parts. 
The details of the mirror testing system are shown in Figure 5. The PVDF laminated membrane mirror is carefully stretched by hand in all directions to remove any wrinkles that may be present and the mirror is then clamped by two aluminum rings on the boundary. The membrane mirror is supported and fixed on an optical bench by four aluminum supports. Three laser probe heads are hung vertically on the test mirror to measure the displacement of three points on the mirror. The three points, i.e., the centers of each PVDF actuator, which form a triangle, are used to represent the approximate surface deformation of the mirror in this experiment. A ceramic heater connected to a temperature controller is placed under the center of the mirror to serve as an external thermal load. The temperature of the ceramic heater can be adjusted from room temperature to $200{ }^{\circ} \mathrm{C}$. Note that the ceramic heater cannot be seen in Figure 5 from this perspective and all leads have been removed from the PVDF actuators.

Three PVDF patches are laminated on the nonreflective side of the mirror by rubber cement as in-plane actuators. Ideally, it is assumed that the PVDF patches are fixed tightly onto the mirror and there is no relative displacement between the two layers. The layout of the PVDF actuators is shown in Figure 5. The angle between each actuator is $120^{\circ}$. The dimensions of the membrane mirror and the PVDF patches are given in Table 1.

The properties of the polyimide substrate and PVDF films are listed in Table 2 and Table 3.

\section{Implementation and Results}

From the mathematical model established previously, we can find that the transverse deflection of the mirror is caused by the piezoelectric forces and moments induced by the PVDF actuators. However, because of the

Table 1 Dimensions of mirror and actuators

\begin{tabular}{llll}
\hline $\begin{array}{l}\text { Radius } \\
R_{m} / \mathrm{m} \\
\text { (mirror) }\end{array}$ & $\begin{array}{l}\text { Inner radius } \\
R_{\mathbf{1}} / \mathrm{m} \\
\text { (actuators) }\end{array}$ & $\begin{array}{l}\text { Outer radius } R_{\mathbf{2}} / \mathrm{m} \\
\text { (actuators) }\end{array}$ & $\begin{array}{l}\text { Arc length } \\
\boldsymbol{\theta} /\left(^{\circ}\right) \\
\text { (actuators) }\end{array}$ \\
\hline 0.1 & 0.03 & 0.07 & 50 \\
\hline
\end{tabular}

Table 2 Polyimide substrate material properties

\begin{tabular}{ll}
\hline Parameter & Value \\
\hline Thickness $h_{s} / \mathrm{mm}$ & 0.1 \\
Young's modulus $E_{s} / \mathrm{MPa}$ & 2170 \\
Poisson's ratio $v_{s}$ & 0.34 \\
Density $\rho_{s} /\left(\mathrm{kg} / \mathrm{m}^{3}\right)$ & 1434 \\
Thermal expansion coefficient $a_{s} /\left({ }^{\circ} \mathrm{C}^{-1}\right)$ & $2.9 \times 10^{-5}$ \\
\hline
\end{tabular}

Table 3 PVDF film material properties

\begin{tabular}{ll}
\hline Parameter & Value \\
\hline Thickness $h_{p} / \mathrm{mm}$ & 0.1 \\
Young's modulus $E_{p} / \mathrm{MPa}$ & 2500 \\
Poisson's ratio $v_{p}$ & 0.35 \\
Density $\rho_{p} /\left(\mathrm{kg} / \mathrm{m}^{3}\right)$ & 1780 \\
Thermal expansion coefficient $a_{p} /\left({ }^{\circ} \mathrm{C}^{-1}\right)$ & $1.53 \times 10^{-4}$ \\
Piezoelectric constant $d_{31} /(\mathrm{pC} / \mathrm{N})$ & 17 \\
Piezoelectric constant $d_{32} /(\mathrm{pC} / \mathrm{N})$ & 5 \\
\hline
\end{tabular}

nonlinearity and complexity of the model, it is difficult to obtain analytical solutions and to establish a closedloop feedback control system for the model. In this section, a simplified method based on the influence function matrix (IFM) of the system is used to adaptively control the shape of the membrane mirror.

\subsection{Influence Function Matrix}

The IFM of the membrane mirror system is a matrix obtained by an experiment based on the proportional error feedback algorithm that can describe the relationship between the input voltage of each actuator and surface shape of the mirror under a particular layout of the PVDF actuators. In this method, the driving voltage of each actuator can be calculated in real time with laser displacement sensors that measure the surface in high frequency, and therefore, closed-loop control is achieved. Studies conducted in AFIT [29], PSU [35], and CIT [36] have proved that when implementing small membrane deflection control with PVDF actuators, the control effect can be deemed approximately linear, i.e., the transverse deformation of the mirror is assumed basically proportional to the voltage applied to the PVDF patches. Hence, voltages ranging from $-200 \mathrm{~V}$ to $200 \mathrm{~V}$ at $50 \mathrm{~V}$ increments are applied to actuator No. 1 to validate this basic assumption. The deflection of reference point 1 is measured and recorded by the laser displacement sensor and then plotted in Figure 6. The green stars are the original data collected by the displacement sensor under each voltage and the red line denotes the fitted straight line. The linear relationship between the voltage and membrane deformation can be observed in Figure 6 .

With the linear assumption above, the IFM of the mirror can be obtained by experimental identification. The centers of the three PVDF patches are selected as reference measuring positions of the laser displacement sensors. The three points form a triangle, which is used to approximately represent the surface shape of the mirror. Consider each displacement sensor signal as a static response vector of the mirror and put it in matrix $\boldsymbol{R}$. Then the mirror static system could be expressed as 


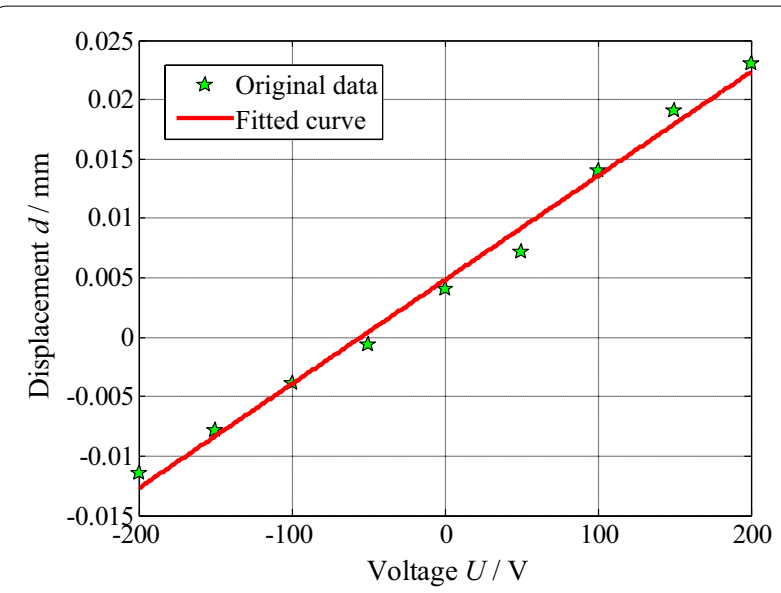

Figure 6 Linear actuation effect assumption test

$$
A_{3 \times 3} X_{3 \times 3}=R_{3 \times 3},
$$

where

$$
\boldsymbol{R}_{3 \times 3}=\left(\begin{array}{lll}
\boldsymbol{w}_{1} & \boldsymbol{w}_{2} & \boldsymbol{w}_{3}
\end{array}\right)_{3 \times 3} .
$$

$\boldsymbol{A}$ is the unknown mirror system and $\boldsymbol{X}$ is an identity $3 \times 3$ matrix corresponding to a one-volt application to each of the actuators. The vectors $\boldsymbol{w}_{1}, \boldsymbol{w}_{2}$, and $\boldsymbol{w}_{3}$ represent the displacement response of the mirror for a one-volt application to actuators 1, 2, and 3 respectively. Basically, $\boldsymbol{A}=\boldsymbol{R}$. For the control problem, where a desired surface $\boldsymbol{w}$ represented by three displacement data is desired, the problem to be solved is

$$
A_{3 \times 3} v_{3 \times 1}=w_{3 \times 1},
$$

where $v$ represents the control voltage inputs. By simplifying the expression, we obtain

$$
\boldsymbol{v}_{3 \times 1}=\boldsymbol{K}_{3 \times 3} \boldsymbol{w}_{3 \times 1},
$$

where $K$ is the IMF of the mirror and is determined using

$$
\boldsymbol{K}=\boldsymbol{A}^{-1} \text {. }
$$

Table 4 Displacement induced by actuator No. 1 ( $\mathrm{mm})$

\begin{tabular}{llll}
\hline Voltage (V) & Sensor No. 1 & Sensor No. 2 & Sensor No. 3 \\
\hline+200 & 0.0129 & -0.0059 & -0.0069 \\
-200 & -0.0149 & 0.0046 & 0.0034 \\
\hline
\end{tabular}

Note that the IFM $\boldsymbol{K}$ acted on the error signal in the feedback path, where the error signal is defined as

$$
\boldsymbol{w}=\boldsymbol{w}_{\text {desired }}-\boldsymbol{w}_{\text {measured }} \cdot
$$

Table 5 Displacement induced by actuator No. 2 (mm)

\begin{tabular}{llll}
\hline Voltage (V) & Sensor No. 1 & Sensor No. 2 & Sensor No. 3 \\
\hline+200 & -0.0160 & 0.0020 & -0.0034 \\
-200 & -0.0096 & -0.0208 & -0.0086 \\
\hline
\end{tabular}

Table 6 Displacement induced by actuator No. $3(\mathrm{~mm})$

\begin{tabular}{llll}
\hline Voltage (V) & Sensor No. 1 & Sensor No. 2 & Sensor No. 3 \\
\hline+200 & -0.0179 & 0.0231 & 0.0093 \\
-200 & -0.0120 & 0.0338 & -0.0098 \\
\hline
\end{tabular}

Voltages of $\pm 200 \mathrm{~V}$ are applied to actuators Nos. 1-3 in sequence and the displacements of the mirror in each point are recorded in Tables 4, 5 and 6 .

Then, the IFM $K$ can be calculated using the data in Tables 4, 5 and 6 and Eqs. (56) and (59).

$$
\boldsymbol{K}=\left(\begin{array}{ccc}
18.53 & 3.45 & 7.66 \\
11.73 & 17.74 & 13.56 \\
6.80 & -2.97 & 21.38
\end{array}\right)(\mu \mathrm{m})
$$

\subsection{Membrane Shape Tracking Experiments}

To verify the correctness of the calculated value of $\boldsymbol{K}$, a series of membrane shape tracking tests were performed. Different signals including sinusoidal, square, and linear waves are input into the control system as reference shape of the mirror and three outputs of the mirror are tracked simultaneously.

For displacement sensor No. 1, a sinusoidal signal is input as the desired shape:

$$
y(t)=10 \sin (2 \pi t) .
$$

For displacement sensor No. 2, a square signal is input as the desired shape:

$$
y(t)=\left\{\begin{array}{r}
10, n \leq t<n+0.5, \\
-10, n+0.5 \leq t<n+1,
\end{array} \quad n=0,1,2, \cdots\right.
$$

For displacement sensor No. 3, a linear zero signal is input as the desired shape:

$$
y(t)=0 \text {. }
$$

The membrane shape tracking tests for the three different desired shape signals are shown in Figure 7(a), (b), and (c) respectively. The black line indicates the command input (i.e., the shape signal to be tracked) and the red dashed line is the closed-loop mirror displacement response. From Figure 7, it can be observed that the shape signals are tracked with recognizable accuracy, which proves the effectiveness of the IFM for the membrane mirror shape control. 


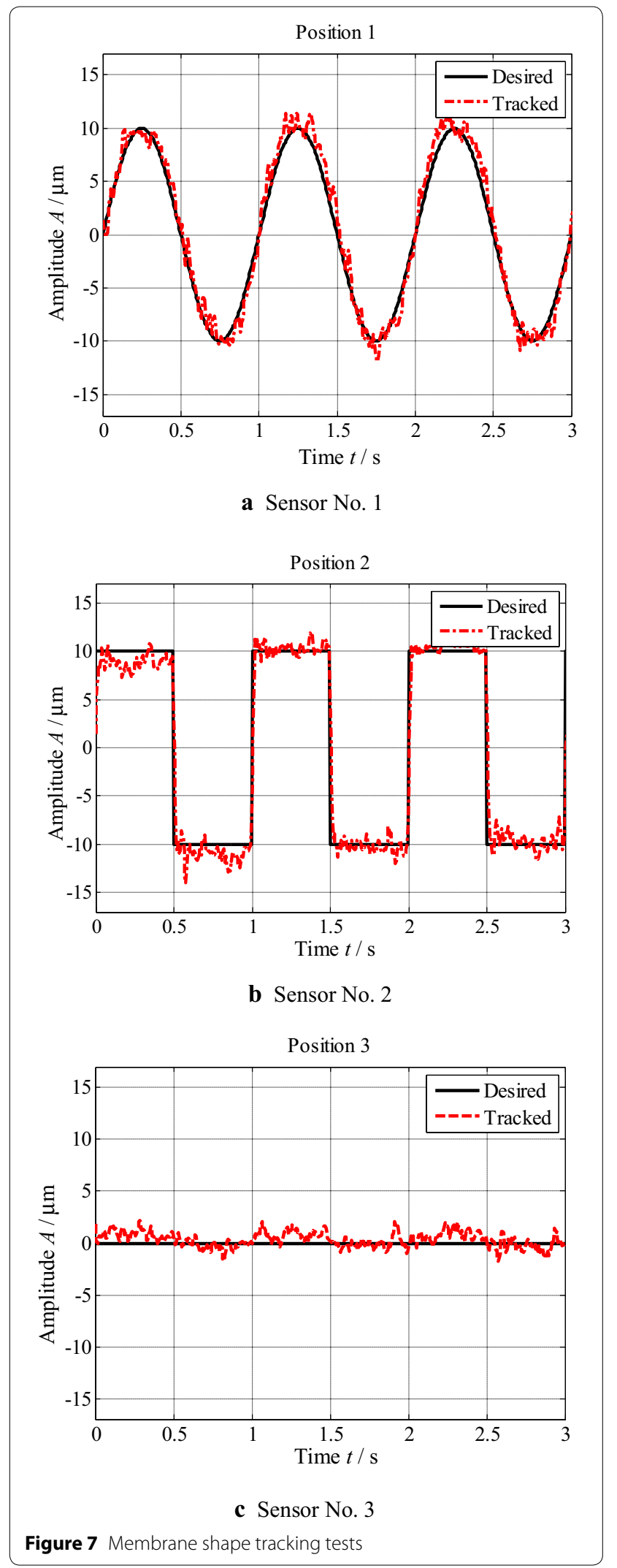

\subsection{Adaptive Shape Control for Thermal Deformation}

Thermal loads are the main cause of membrane distortion in outer space. In this section, we describe the investigation of the thermal deformation of the membrane mirror induced by a ceramic heater. The room temperature is approximately $25^{\circ} \mathrm{C}$ during the experiment. The ceramic heater was started and the temperature controller was set at $40{ }^{\circ} \mathrm{C}$. It is obvious that the membrane mirror will thermally deform when heated. A linear signal $y(t)=0$ is input into the controller as the desired shape for sensor No. 1 to 3 to track so that the surface deviation can be decreased. The controller starts to work at the eighth second. The time-varying signals of three displacement sensors are shown in Figure 8(a), (b), and (c). The black line represents the desired shape signal and the red dashed line denotes the controlled transverse deformation of the mirror.

Figure 8 shows that mirror deformation occurs because of the thermal load and it increases gradually as the temperature rises. The deviations measured by the three displacement sensors range from $15 \mu \mathrm{m}$ to $20 \mu \mathrm{m}$. However, the deviations in all three locations improve remarkably and rapidly after the controller begins to work at the eighth second. The residual deformation is controlled to the micron level. These experimental results confirm the effectiveness of the proposed membrane shape control strategy and show a satisfactory real-time performance.

\section{Conclusions}

(1) A mathematical model of a piezoelectric laminated membrane mirror in multiple fields is established. With specific Lamé parameters and radii of curvature, the governing equations of the mirror are simplified. The strain and stress of the mirror are calculated, respectively, from which the membrane force and bending moment expressions are derived.

(2) A new transverse dynamic/static equation and a compatibility equation including piezoelectric and thermal effects are obtained. The dynamic and static behaviors of the membrane mirror are analyzed using these newly derived equations.

(3) Owing to the complexity of the mathematical model, it is difficult to obtain analytical solutions for feedback control on the shape of the mirror. A surface shape control method based on IFM is investigated.

(4) The IFM of the mirror is identified through experiments, and shape tracking experiments are then conducted. Different wave signals are input into the control system as reference shape and outputs (transverse deflection) of the mirror are monitored to track the desired membrane deformation with recognizable accuracy. 


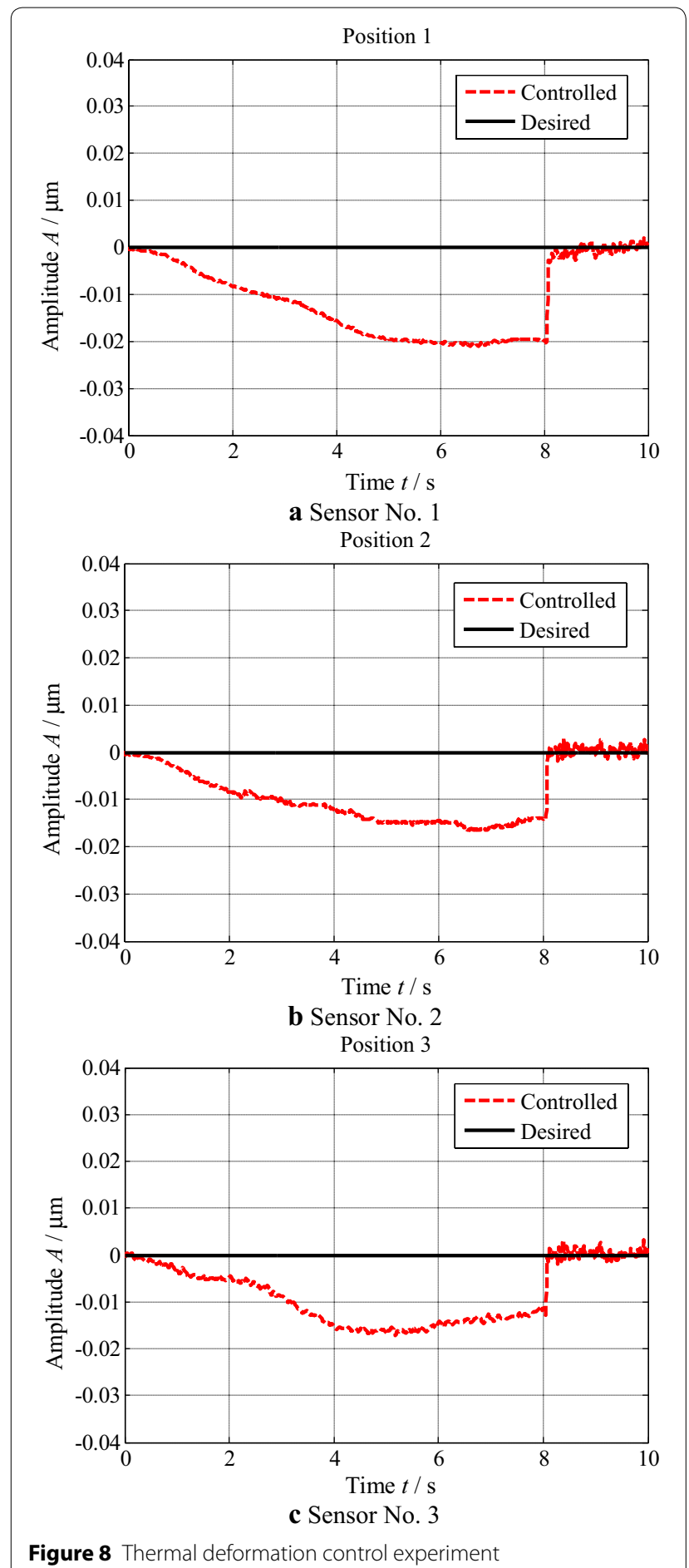

(5) A ceramic heater is placed under the center of the mirror to serve as a thermal load, and the surface shape of the membrane is controlled by inputting a zero signal as the design deformation. It is demonstrated that with this developed control method, the thermal deformation of the mirror can be controlled effectively and rapidly.

\section{Authors' Information}

Yi-Fan Lu, born in 1989, is currently a PhD candidate at Research Center of Aerospace Mechanism Control, Harbin Institute of Technology, China, majoring in mechatronics engineering. His research interests include sensing and active control of smart materials and structures. His research topic is now focusing on quasi-static and dynamic control of membrane plate and shell structures. E-mail: luyifanluyifan@126.com.

Hong-Hao Yue, born in 1978, is currently a professor and a PhD candidate supervisor at Research Center of Aerospace Mechanism Control, Harbin Institute of Technology, China. His main research interests include mechatronics engineering and active vibration control of smart structures. E-mail: block@ hit.edu.cn

Zong-Quan Deng, born in 1966, is currently a professor and a PhD candidate supervisor at Research Center of Aerospace Mechanism Control, Harbin Institute of Technology, China. His main research interests include space mechanism control and mobile robot in special environment. E-mail: dengzq@hit.edu.cn.

Hornsen Tzou, is currently a professor and a PhD candidate supervisor at College of Aerospace Engineering, Nanjing University of Aeronautics and Astronautics, China. His research interests include smart structures and structronic systems and distributed sensing and control of plates and shells. E-mail: hstzou@nuaa.edu.cn.

\section{Author details}

${ }^{1}$ School of Mechatronics Engineering, Harbin Institute of Technology, Harbin 150001, China. ${ }^{2}$ College of Aerospace Engineering, Nanjing University of Aeronautics and Astronautics, Nanjing 210016, China.

\section{Authors' contributions}

$Y L$ conceived and designed the study. $Y L$ and $H Y$ performed the experiments. YL wrote the paper. ZD and HT reviewed and edited the manuscript. All authors read and approved the final manuscript.

\section{Acknowledgements}

This research is supported by the National Natural Science Foundation of China (Grant No. 51175103) and Self-Planned Task of State Key Laboratory of Robotics and System (HIT) (Grant No. SKLRS201301B).

\section{Competing interests}

The authors declare that they have no competing interests.

\section{Ethics approval and consent to participate}

Not applicable.

\section{Publisher's Note}

Springer Nature remains neutral with regard to jurisdictional claims in published maps and institutional affiliations.

Received: 20 July 2016 Accepted: 14 January 2018

Published online: 28 February 2018

\section{References}

1. R Angel. Future very large space telescopes. Space 2000 Conference and Exposition. Long Beach, United states, September 19-21, 2000: 5304.

2. D Gorinevsky, TT Hyde. Adaptive membrane for large lightweight space telescopes. Highly Innovative Space Telescope Concepts, Waikoloa, USA, March 5-8, 2002: 330-338.

3. Y F Lu, H H Yue, Z Q Deng, et al. Research on active control for thermal deformation of precise membrane reflector with boundary SMA actuators. ASME 2014 International Mechanical Engineering Congress and Exposition, Montreal, Canada, November 14-20, 2014: V04BT04A061.

4. E Yoo, J Roh, J Han. Wrinkling control of inflatable booms using shape memory alloy wires. Smart Materials and Structures. 2007, 16(2): 340. 
5. H Furuya, Y Miyazaki, Y Akutsu. Experiments on static shape control of one-dimensional creased membrane by piezoelectric films. 43rd Structures, Structural Dynamics, and Materials Conference, Denver, United states, April 22-25, 2002: 1377

6. M J Shepherd, R G Cobb, W P Baker. Low-order actuator influence functions for piezoelectric in-plane actuated tensioned circular deformable mirrors. Smart Structures and Materials 2006: Modeling, Signal Processing, and Control, San Diego, United states, February 27-March 2, 2006: 61660E-61660E-12.

7. H Fang, M J Pattom, K Wang, et al. Shape control of large membrane reflector with PVDF actuation. 48th AIAA/ASME/ ASCE/AHS/ASC Structures, Structural Dynamics, and Materials Conference, Waikiki, United states, April 23-26, 2007: 1842.

8. STung, S R Witherspoon, L A Roe, et al. A MEMS-based flexible sensor and actuator system for space inflatable structures. Smart Materials and Structures, 2001, 10(6): 1230.

9. A Doosthoseini, A Salehian, M Daly. Analysis of wrinkled membranes bounded with macro-fiber composite (MFC) actuators. ASME 2010 Conference on Smart Materials, Adaptive Structures and Intelligent Systems. American Society of Mechanical Engineers, Philadelphia, United states, September 28 - October 1, 2010: 583-588.

10. E Fleurent, TE Pollock, W Su, et al. Wrinkle localization in membrane structures patched with macro-fiber composite actuators: Inflatable space antenna applications. Journal of Intelligent Material Systems and Structures, 2014, 25(15): 1978-2009.

11. J Su, TXu, S Zhang, et al. A hybrid actuation system (HYBAS) and aerospace applications. 2005 Materials Research Society Fall Meeting, Boston, United States, November 28-December 1, 2005, 888: 27-34.

12. A K Maji, M A Starnes. Shape measurement and control of deployable membrane structures. Experimental Mechanics, 2000, 40(2): 154-159.

13. S Errico, J R Angel, B L Stamper, et al. Stretched Membrane with Electrostatic Curvature (SMEC) Mirrors: A new technology for large lightweight space telescopes. Highly Innovative Space Telescope Concepts, Waikoloa, USA, August 22-23, 2002: 356-364.

14. F Peng, Y Hu, A Ng. Testing of inflatable-structure shape control using genetic algorithms and neural networks. AIAA Journal, 2007, 45(7): 1771-1774.

15. F Peng, Y Hu, A Ng. Testing of membrane space structure shape control using genetic algorithm. Journal of Spacecraft and Rockets, 2006, 43(4): 788-793.

16. F J Peng, Y R Hu, A Ng. Development of GA-based control system for active shape control of inflatable space structures. 2005 IEEE International Conference on Control Applications (CCA), Toronto, Canada, August 28-31, 2005: 577-582.

17. F Peng, X Jiang, Y Hu, et al. Application of SMA in membrane structure shape control. IEEE Transactions on Aerospace and Electronic Systems, 2009, 45(1): 85-93.

18. X Wang, W Zheng, Y Hu. Active flatness control of space membrane structures using discrete boundary SMA actuators. IEEE/ASME International Conference on Advanced Intelligent Mechatronics, AIM 2008, Xi'an, China, July 2-5, 2008: 1108-1113.

19. X Wang, C Sulik, W Zheng, et al. Thermo-mechanical analysis of thin membranes and application in active flatness control design. Industrial and Commercial Applications of Smart Structures Technologies 2008, San Diego, United States, March 10-11, 2008: 69300H-69300H-12.
20. X Wang, W Zheng, Y R Hu. An experimental study on the interaction of thermal loading and mechanical loading in membrane structures. Strain, 2011, 47: 493-504

21. J Shan, R Orszulik, M Girin, et al. Flatness measurement and active control for a membrane structure. 2012 IEEE International Conference on Mechatronics and Automation (ICMA), Chengdu, China, August 5-8, 2012: 1062-1067.

22. C H Jenkins, D K Marker. Surface precision of inflatable membrane reflectors. Transactions-American Society of Mechanical Engineers Journal of Solar Energy Engineering, 1998, 120: 298-305.

23. C H Jenkins, S M Faisal. Thermal load effects on precision membranes. Journal of Spacecraft and Rockets, 2001, 38(2): 207-211.

24. C H Jenkins, D M Fitzgerald, X Liu. Wrinkling of an inflated membrane with thermo-elastic boundary restraint. 41st AIAA Structures, Structural Dynamics, and Materials Conference, Atlanta, USA, April 3-6, 2000: 76-83.

25. C H Jenkins, D K Marker, J M Wilkes. Improved surface accuracy of precision membrane reflectors through adaptive rim control. 39th AIAA/ASME/ ASCE/AHS/ASC Structures, Structural Dynamics, and Materials Conference and Exhibit. Long Beach, USA, April 20-23, 1998: 2302-2308.

26. J R Blandino, J D Johnston, J J Miles, et al. Thin film membrane wrinkling due to mechanical and thermal loads. 19th AlAA Applied Aerodynamics Conference, Anaheim, United States, June 11-14, 2001: 1345.

27. J R Blandino, J D Johnston, J J Miles, et al. The effect of asymmetric mechanical and thermal loading on membrane wrinkling. 43rd Structures, Structural Dynamics and Materials Conference, Denver, United States, April 22-25, 2002: 1282-1292.

28. J Hornig, H Schoop, U Herbrich. Wrinkling analysis of thermoelastic membranes. Technische Mechanik, 2006, 26(1): 33-43.

29. M J Shepherd. Lightweight in-plane actuated deformable mirrors for space telescopes. Air Force INST of Tech Wright-Patterson AFB OH School of Engineering and Management, 2006.

30. M Laslandes, K Patterson, S Pellegrino. Optimized actuators for ultrathin deformable primary mirrors. Appl Opt., 2015, 54(15): 4937-4952.

31. P Rausch, S Verpoort, U Wittrock. Unimorph deformable mirror for space telescopes: environmental testing. Optics Express., 2016, 24(2): 1528.

32. H Tzou. Piezoelectric shells: distributed sensing and control of continua Kluwer Academic Publishers, 1993.

33. W Soedel. Vibrations of shells and plates. CRC Press, 2004.

34. A H Nayfeh, P F Pai. Linear and nonlinear structural mechanics. John Wiley \& Sons, Inc., 2008.

35. J R Hill. High precision surface control of flexible space reflectors. The Pennsylvania State University, 2011.

36. KD Patterson. Lightweight deformable mirrors for future space telescopes. California Institute of Technology, 2014.

\section{Submit your manuscript to a SpringerOpen ${ }^{\circ}$ journal and benefit from:}

- Convenient online submission

- Rigorous peer review

- Open access: articles freely available online

- High visibility within the field

- Retaining the copyright to your article

Submit your next manuscript at springeropen.com 